\title{
Synaptic vesicle pools: an update
}

\author{
Annette Denker ${ }^{1,2 *}$ and Silvio O. Rizzoli ${ }^{1 *}$ \\ 1 European Neuroscience Institute, DFG Center for Molecular Physiology of the Brain, Göttingen, Germany \\ 2 International Max Planck Research School Molecular Biology, Göttingen, Germany
}

\author{
Edited by: \\ Robert Renden, Heidelberg University, \\ Germany; UCB Pharma SA, Belgium \\ Reviewed by: \\ David Zenisek, Yale University, USA \\ Thomas Kuner, University of \\ Heidelberg, Germany \\ *Correspondence: \\ Annette Denker and Silvio O. Rizzoli, \\ European Neuroscience Institute, \\ STED Microscopy of Synaptic Function, \\ Grisebachstr. 5, 37077 Göttingen, \\ Germany. \\ e-mail: srizzol@gwdg.de; adenker@ \\ gwdg.de
}

During the last few decades synaptic vesicles have been assigned to a variety of functional and morphological classes or "pools". We have argued in the past (Rizzoli and Betz, 2005) that synaptic activity in several preparations is accounted for by the function of three vesicle pools: the readily releasable pool (docked at active zones and ready to go upon stimulation), the recycling pool (scattered throughout the nerve terminals and recycling upon moderate stimulation), and finally the reserve pool (occupying most of the vesicle clusters and only recycling upon strong stimulation). We discuss here the advancements in the vesicle pool field which took place in the ensuing years, focusing on the behavior of different pools under both strong stimulation and physiological activity. Several new findings have enhanced the three-pool model, with, for example, the disparity between recycling and reserve vesicles being underlined by the observation that the former are mobile, while the latter are "fixed". Finally, a number of altogether new concepts have also evolved such as the current controversy on the identity of the spontaneously recycling vesicle pool.

Keywords: synaptic vesicle pools, spontaneous release, surface pool, super-pool, vesicle mobility, vesicle recycling
The concept that synaptic vesicles are not all functionally equivalent has been introduced almost fifty years ago by the investigations of Birks and MacIntosh (1961) in cat sympathetic ganglia: some vesicles were more easily released than others. In the meantime, the concept has been expanded to essentially all major synaptic preparations, including synapses as different as the Drosophila larval neuromuscular junction (NMJ), goldfish retinal bipolar cells, the frog NMJ, the mammalian calyx of Held (a giant synapse in the auditory system), and cultured hippocampal synapses. For all these preparations, three major synaptic vesicle pools have been proposed: a readily releasable pool (RRP), a recycling pool and a reserve pool (Rizzoli and Betz, 2005). The recycling pool consists of the synaptic vesicles which recycle upon moderate (physiological) stimulation, typically about $10-20 \%$ of all vesicles. The RRP consists of the ("lucky") recycling pool vesicles which find themselves docked and primed for release; these are the vesicles released immediately upon stimulation. Finally, the reserve pool hosts vesicles which are reluctant to release, and which are therefore only recruited upon high-frequency stimulation, after depletion of the recycling pool (Figures 1A,B).

The synaptic vesicle pool field has advanced substantially in recent years. We focus on several recent findings throughout this review. First, the concept of three different vesicle pools has been originally developed almost exclusively from high-frequency stimulation experiments. It is therefore worth discussing whether the three-pool paradigm also holds true under lower (more physiological) stimulation conditions. Second, several additional vesicle "pools" have been suggested (largely overlapping with the previously described pools): the spontaneously releasing pool, suggested to host vesicles responsible for spontaneous release (Sara et al., 2005); the stranded vesicle pool, containing vesicle proteins corresponding to fused synaptic vesicles (Wienisch and Klingauf, 2006); and the "super-pool", composed of vesicles which are exchanged between neighboring synapses at a high rate (Darcy et al., 2006). Third, while several new concepts and ideas were introduced within the last few years, the long-lasting debate on the mode of synaptic vesicle recycling is still not resolved, with evidence presented for each of the models (kissand-run, clathrin-mediated endocytosis, endosomal recycling, bulk endocytosis; see also Rizzoli and Jahn, 2007). Finally, the inherent question on how the different vesicle pools maintain their respective identities throughout recycling has not yet been answered.

\section{SYNAPTIC VESICLE POOLS UNDER DIFFERENT STIMULATION PARADIGMS}

An intuitive model of pool structure originally assumed that pool affiliation depended on vesicle localization within the terminal, with the RRP located directly at the active zone, the recycling pool just behind, and the reserve pool farther away from the active zone (Figure 1A). The first doubts were cast on this model from a nonsynaptic perspective, in studies of chromaffin cells. These cells employ an exocytotic machinery which is similar to that of conventional synapses, although the vesicles they release are very different from those of most synapses (dense-core vesicles, rather than small clear-core, neurotransmitter-filled vesicles). A pool model derived from experiments with laser photolysis of caged-calcium (which raises the calcium inside the cells instantly, and allows monitoring the ensuing release by capacitance recording) indicated that at least three pools participate in release: an RRP (providing a fast burst of release), a slowly releasable one (resulting in a slower release burst, which is nevertheless completed in less than one second after the calcium increase), and finally an unprimed pool, which releases slowly and constantly over many seconds (reviewed by Rettig and Neher, 2002). Clearly, no difference in positioning (at the plasma membrane) could explain the different release rates, with the calcium rise not linked to entry through calcium channels in the plasma membrane. 
Such experiments were also performed in synapses such as the calyx of Held (Schneggenburger et al., 1999; Schneggenburger and Neher, 2000; Bollmann et al., 2000; reviewed by Schneggenburger et al., 2002; Schneggenburger and Neher, 2005). The calcium rise caused the release of an RRP of definite size, although, as in chromaffin cells, this pool may not be completely homogeneous. Nevertheless, most vesicles contained within the RRP were released within a few milliseconds, so could only have been docked at the active zones (i.e., in the vicinity of the calcium channels).

However, morphological studies of pool localization indicated a strong intermixing between the reserve and recycling pools. Therefore, while one of the components of the intuitive model

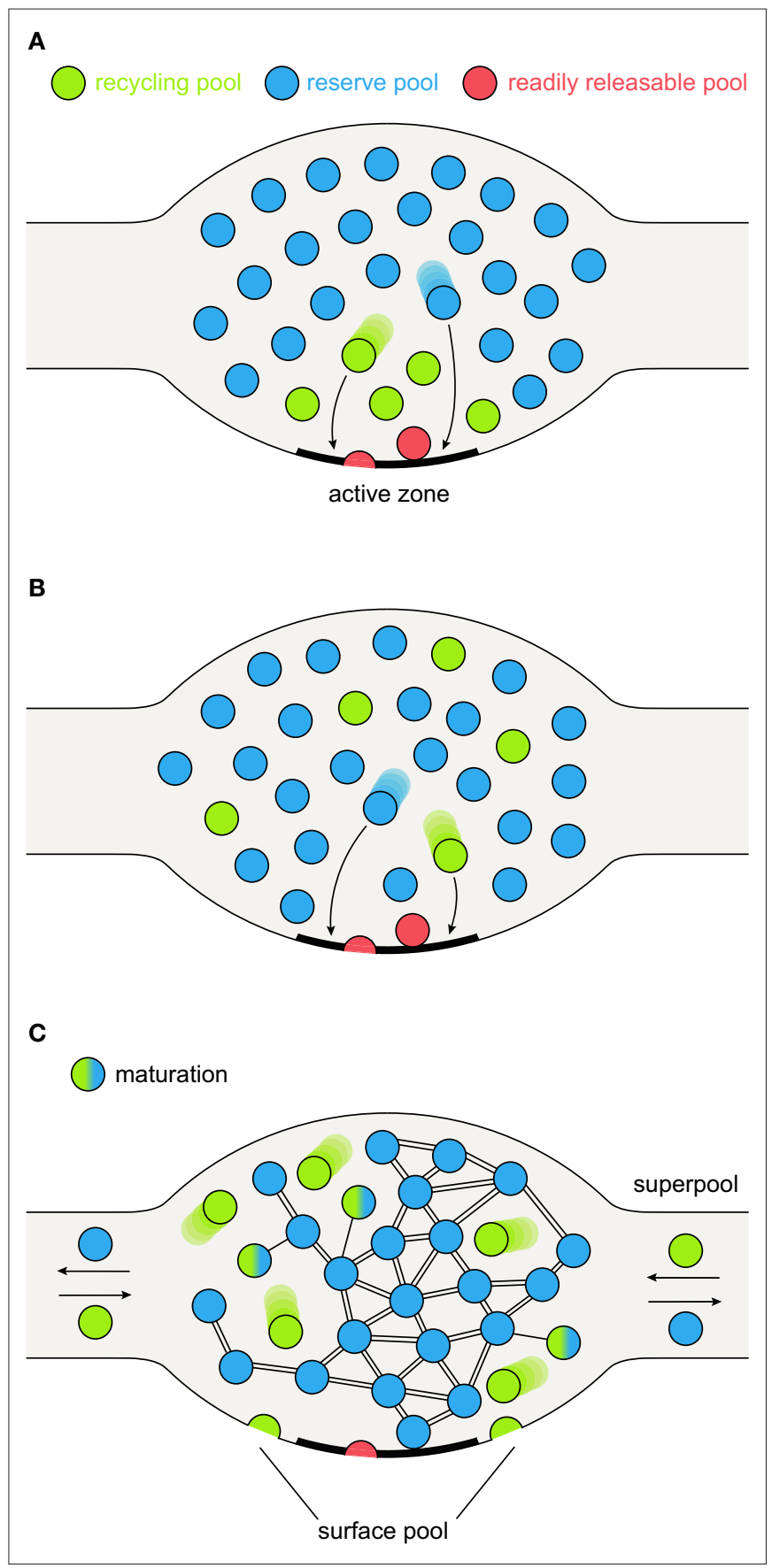

remains valid (the RRP vesicles have a privileged position, docked at active zones), all other vesicles are scattered throughout the synapse (Figure 1B) (Rizzoli and Betz, 2005). Note that this implies another important difference versus the intuitive model: not all synaptic vesicles docked at the active zones will necessarily be rapidly releasable (or even releasable). In the frog NMJ the active zones were largely occupied by vesicles which did not (and likely could not) release during physiological stimulation (Rizzoli and Betz, 2004), with a similar phenotype in Drosophila NMJs (Denker et al., 2009).

While closer to the functional morphology of the synapse, this model is nevertheless still derived from strong stimulation experiments (typically $>10 \mathrm{~Hz}$ for many seconds). Undoubtedly, in many preparations stimulation triggers first the release of "fast" vesicles (the RRP), followed by slower-releasing ones (recycling pool/ reserve pool; for example in human intercostal muscle, Elmqvist and Quastel, 1965; the Drosophila NMJ, Delgado et al., 2000; and the rat calyx of Held, Schneggenburger et al., 1999; Scheuss et al., 2002). However, defining vesicle pools based on high-frequency stimulation poses one major problem: stimulation frequencies of $10-100 \mathrm{~Hz}$ are not physiologically relevant for conventional synapses, especially when applied for many seconds or minutes.

Strong stimulation provided a seemingly irrefutable proof for the presence of the reserve pool, as it often left a proportion of the vesicles unreleased (a "resting" reserve pool). Resting vesicles (at least $\sim 50 \%$ of all vesicles) have been observed, for example, in cultured hippocampal neurons (Harata et al., 2001a,b; FernandezAlfonso and Ryan, 2008; Opazo et al., 2010), the mouse NMJ

FIGURE 1 | Synaptic vesicle pool models. (A) The classical model of three distinctly localized synaptic vesicle pools. The readily releasable pool (RRP; depicted in red) consists of the vesicles docked at the active zone and primed for release. After depletion of the RRP, the recycling pool vesicles come into play (green): these vesicles were thought to be located directly behind the RRP. Under moderate stimulation conditions, they are recruited to the active zone (left arrow) and released. Very high stimulation causes the depletion of the recycling pool and recruits the reserve pool vesicles (blue) from areas even further away from the active zone (right arrow). (B) A pool model taking into account the spatial intermixing of vesicles (Rizzoli and Betz, 2005). In contrast to the previous model, in which localization within the nerve terminal determines release kinetics and consequently pool affiliation, here the recycling and reserve pool vesicles are thought to be spatially (but not functionally) intermixed. Upon arrival of an action potential, RRP vesicles (which are in this model only the "lucky" recycling pool vesicles finding themselves docked and primed at the active zone) are released first, followed by release of recycling pool vesicles (right arrow). As above, continuous stimulation at high-frequency results in recycling pool depletion and recruitment of reserve pool vesicles (left arrow). (C) A new pool model implementing recent findings. As above, recycling and reserve pool vesicles are thought to be spatially intermixed, but display different mobilities: recycling pool vesicles are highly mobile; the movement of reserve pool vesicles is restricted by binding to some scaffolding molecule. With time, recycling pool vesicles can "mature" into reserve pool vesicles, by binding the scaffolding molecule(s) and integrating into the vesicle cluster, as indicated by the green-blue intermediate forms. These are not connected to the cluster as tightly as the reserve pool vesicles (indicated by single or double bonds). Recycling pool vesicles reach the active zone, due to their permanent mobility; stimulation does not "move" them toward the active zone, it just allows them to fuse. Furthermore, the surface pool of fused vesicles is indicated; they would be endocytosed to form part of the recycling pool (see main text). The frequent exchange of both recycling and reserve vesicles between synapses forms what has been termed a super-pool (see main text). 
(Wyatt and Balice-Gordon, 2008) and the rat calyx of Held (de Lange et al., 2003). In other preparations virtually all vesicles recycle upon strong stimulation, as in the frog or Drosophila NMJs (Richards et al., 2000; Rizzoli and Betz, 2004; Akbergenova and Bykhovskaia, 2007, 2009; Denker et al., 2009). Nevertheless, also in these preparations a distinction between recycling and reserve pool vesicles could be made when employing milder stimulation (still somewhat above the physiological range of the preparations, as for high potassium depolarization or $5 \mathrm{~Hz}$ stimulation): a large (50-80\%) proportion of the vesicles remained unused (Kuromi and Kidokoro, 1998, 1999, 2000, 2002; Richards et al., 2003).

How do these differences between the pools fare under physiological frequencies of stimulation? Importantly, pool-defining experiments have been typically performed not only at high intensities of stimulation, but also over a relatively short timeon the order of minutes. Using low frequencies of stimulation over many minutes or hours (as in a living organism) has resulted in a somewhat different picture: Ceccarelli et al. (1972) indicated that nearly all vesicles had undergone recycling at least once during 6-8 h of continuous $2 \mathrm{~Hz}$ stimulation in the frog NMJ. In the same preparation, vesicles that were fluorescently labeled by styryl dye uptake during recycling at high frequencies $(10 \mathrm{~Hz}$; thereby involving both recycling and reserve pool vesicles), could subsequently be unlabeled during $30 \mathrm{~min}$ stimulation at much lower frequency $(2 \mathrm{~Hz})$ (Betz and Henkel, 1994). Conversely, when the recycling pool vesicles were specifically labeled in the frog NMJ by a short $30 \mathrm{~Hz}$ stimulation pulse, $30 \%$ of them became reluctant to release within several minutes of rest, i.e., they lost the recycling pool status (Rizzoli and Betz, 2004). In hippocampal cultures, stimulation at $0.2 \mathrm{~Hz}$ for several minutes released what seems to be the majority of the vesicles, despite their reluctance to release upon stronger stimulation (Ikeda and Bekkers, 2009). In the temperature-sensitive Drosophila dynamin mutant shibire (Koenig et al., 1983; Koenig and Ikeda, 1989) and in mouse dynamin knockouts (at least for inhibitory synapses, Hayashi et al., 2008) inhibition of vesicle recycling (but not release) depletes large amounts of vesicles upon weak stimulation or spontaneous synapse activity, indicating that the reserve pool will be recruited upon depletion of the recycling pool, even in the absence of intense stimulation.

One is forced to conclude that under physiological stimulation the distinction between the recycling and reserve vesicles is not as evident as under strong stimulation. The intermixing of the two pools is slow enough to allow their differentiation under short stimulation protocols, but it is too fast to see two different pools under slow, long-lasting stimulation.

To account for both high-frequency and physiological stimulation, we propose a further refinement of the pool model: a recycling pool of vesicles does exist, and the recycling pool vesicles docked at active zones form the RRP, as we proposed in the past. However, the differentiation between the recycling and reserve vesicles is not permanent; the recycling vesicles may re-release several times, maintaining the recycling pool status, but they will eventually "mature" into reserve vesicles, over a timescale of minutes or hours (Figure 1C). Docked reserve vesicles will occasionally exocytose, thus acquiring recycling pool status, and replacing the recycling vesicles which have switched to reserve status. The pool behavior upon stimulation is described in Figure 2 (in Figure 2A under strong, unphysiological stimulation, and in Figure 2B under lowfrequency stimulation).

We chose the term "mature" for the reserve pool, as the recycling vesicles turn into reserve vesicles over time, while it is likely that the reserve vesicles cannot simply transform into recycling vesicles. The reserve vesicles only appear to become part of the recycling pool after exo- and endocytosis, i.e., after a vesicle reformation step (see detailed discussion below, under the Section Vesicle Mobility). Therefore, in the life cycle of one vesicle (between endocytosis and re-release), the reserve pool status is the latest step, the "maturity".

In agreement with this model, recently endocytosed (i.e., recycling pool) vesicles have been shown to be quite mobile in hippocampal cultures, whereas this mobility is subsequently lost within the next minutes or hours, as the newly endocytosed vesicles integrate into the general vesicle cluster (Kamin et al., 2010). This change in mobility might well represent the maturation into less mobile reserve pool vesicles (Gaffield et al., 2006). Importantly, this new model of constant exchange between the recycling and reserve pool also implies that a putative molecular tag defining pool affiliation associates with the vesicles only temporarily and/or might display a gradual distribution among the vesicles (this issue will be further discussed below).

A word of caution on the stimulation procedures/stimulation results: while most synapses do not enjoy very high rates of activity under normal circumstances, transmission in sensory systems is much stronger than in conventional synapses. The calyx of Held, functioning in the auditory pathway, is normally active at $25 \mathrm{~Hz}$ in vivo (Hermann et al., 2007, 2009), and can be active also at much higher rates (up to $1000 \mathrm{~Hz}$ !). To cope with such activity, the calyx of Held is a very large synapse, containing tens of thousands of vesicles and hundreds of active zones (Sätzler et al., 2002). Interestingly, it still seems to have a rather small quantal content in vivo (less than 20 vesicles, Lorteije et al., 2009), indicating that very few of its $\sim 550$ active zones (Sätzler et al., 2002) release a vesicle at any one time. In other words, while this synapse is active at very high rates, each of its active zones may act as a "conventional" synapse, in that it releases one vesicle rather rarely. Nevertheless, this synapse is possibly the best suited synapse for high-frequency stimulation studies.

Ribbon synapses are a key component of the sensory pathways, and perhaps they represent the "true" strongly-releasing synapses (see for example reviews by von Gersdorff and Matthews, 1999; Guatimosim and von Gersdorff, 2002; Moser et al., 2006; Nouvian et al., 2006). A specialized "ribbon" structure, made of densely packed proteins and assembled in front of the release sites, is used in guiding vesicles to the release sites (although other interpretations are possible, see Parsons and Sterling, 2003). These synapses release continuously, with vesicles coming from a large (or very large-hundreds of thousands of vesicles) reserve pool. The vesicles are recycled and may eventually find themselves on the ribbons, but selective re-use of a recycling pool is not apparent; it seems more likely that they recycle back to the reserve pool, from which they may bind the ribbons (Rizzoli and Betz, 2005). Finally, all vesicles are mobile in these synapses (in conventional synapses only the recycling pool is mobile, see below), in good agreement with the fact that ribbon synapses lack synapsin, a protein cross-linking vesicles 


\section{A Unphysiological stimulation:}

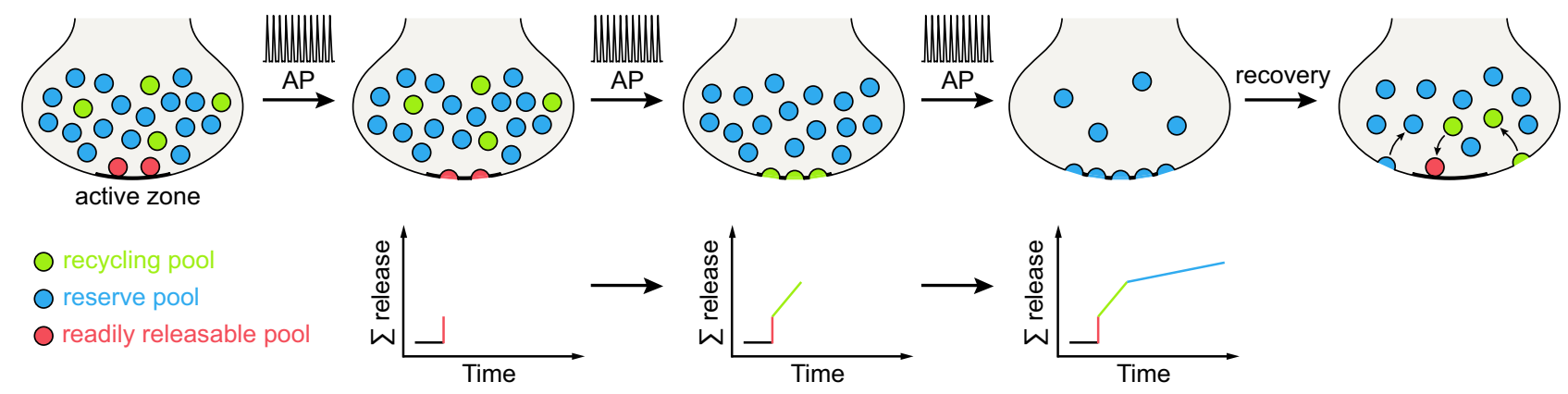

\section{B Physiological stimulation:}
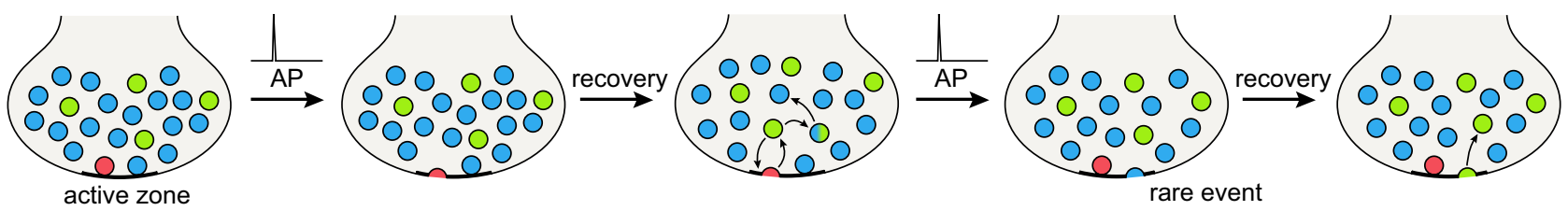

maturation
(recycling to reserve)
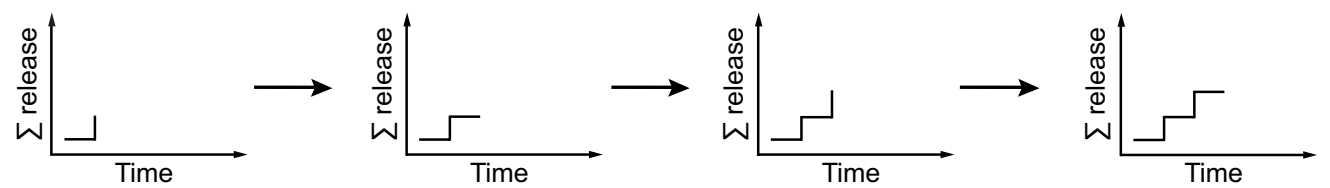

FIGURE 2 | Vesicle release under different stimulation conditions.

(A) Unphysiological stimulation results in release kinetics in accordance with the three pool model (depicted here for spatially intermixed recycling and reserve pool vesicles, Figures 1B,C). Upon arrival of an action potential (AP), the vesicles of the RRP (red) are released immediately. Further stimulation results in the recruitment and release of recycling pool vesicles (green), with release kinetics slower than for the RRP (as the recycling pool vesicles still need to reach the active zone and undergo docking and fusion before exocytosis). When high-frequency stimulation continues, the reserve pool vesicles are finally released (blue), displaying even slower release kinetics than the recycling pool. Therefore, a tri-phasic exocytosis process is observed. When the stimulation has ceased, vesicle components are retrieved from the plasma membrane and the three vesicle pools are reformed (vacant RRP positions are refilled as some recycling pool vesicles dock at the active zone). The lower panels indicate summed release. (B) Physiological stimulation does not allow for the distinction of separate vesicle pools. Upon arrival of an action potential, some of the (RRP) vesicles docked at the active zone are released. However, the mild stimulation paradigm employed allows for a recovery phase, during which these vesicles are retrieved as recycling pool vesicles. They can then either transform into reserve pool vesicles (indicated by the green-blue gradient) or maintain their recycling pool status. Repeated action potentials cause further release of vesicles docked at the active zone. Occasionally, a reserve pool vesicle docked at the active zone will also undergo fusion with the plasma membrane. Upon endocytosis, it will replenish the recycling pool. As no differences in release kinetics can be observed, distinct vesicle pools are not evident from the summed release (indicated by the lower panels). Note that it is also possible that the reserve pool vesicles join the recycling pool before fusion, rather than fusing and becoming recycling vesicles only after endocytosis (not indicated in the graphs); there is currently no evidence for this model that we are aware of (see main text for details). and perhaps involved in their immobilization in clusters (see below). Thus, large size, large vesicle numbers, special "conveyor belts" for release (ribbons), and finally high vesicle mobility all contribute to giving ribbon synapses the ability to release vesicles constantly, under conditions which would be highly unphysiological for conventional synapses.

\section{EVIDENCE FROM pHluorins CONFIRMS THE THREE-POOL MODEL}

One technique which has strongly advanced and expanded within the last few years is the visualization of vesicle exo- and endocytosis by pHluorins (although pHluorins were introduced already in 1998, Miesenbock et al., 1998). pHluorins are pH-sensitive GFP variants, generally fused to the lumenal domain of synaptic vesicle proteins. They are quenched by the internal $\mathrm{pH}$ of the resting vesicle $(\sim 5.6)$, and upon exocytosis (upon the shift to neutral $\mathrm{pH}$ ) they fluoresce brightly (for instance a 20-fold increase in fluorescence was detected for pHluorin fused to the lumenal domain of synaptobrevin, Sankaranarayanan et al., 2000). Upon vesicle retrieval and reacidification, the fluorescence is again quenched, thus allowing the monitoring of both exo- and endocytosis.

However, this dual property of monitoring both exocytosis and endocytosis also reflects one of the major difficulties of this technique: one generally visualizes only a net change in fluorescence, representing a combination of both release and retrieval. Therefore, information on vesicle pools could only be obtained from studies which employed inhibitors of the vesicular proton pump, such as bafilomycin, thereby preventing vesicle reacidification and allowing for the recording of a cumulative exocytotic signal. Interestingly, in cultured hippocampal neurons, a rapid initial rise in fluorescence has been often observed, possibly corresponding to the release of the RRP, followed by slower exocytosis, most likely corresponding 
to the release of the recycling pool (Sankaranarayanan et al., 2000; Fernandez-Alfonso and Ryan, 2004; Fredj and Burrone, 2009); note that this parallels the rapid initial FM destaining followed by a slower release phase observed in most synapses. Importantly, the pHluorin method allows for a very precise estimation of vesicle pool sizes (Fernandez-Alfonso and Ryan, 2008; Fredj and Burrone, 2009): synapses were strongly stimulated to release the total pool of recycling vesicles, and the remaining (unreleased) vesicles were revealed by neutralizing their $\mathrm{pH}$ with ammonia (Roos and Boron, 1981). The proportion of "non-releasable" vesicles was around 50\%, similar to values obtained by investigating native synaptic vesicle proteins by immunostaining (Opazo et al., 2010). When considering such estimates, one should however keep in mind that the ammonia treatment does not reveal the nature of the quenched organelles. Although the simple suggestion is that they represent the "resting" reserve vesicles, other types of vesicles/organelles could also be involved - especially as the pHluorin construct of the plasma membrane protein syntaxin 1 is also found partially in non-releasable organelles (Mitchell and Ryan, 2004).

The results from $\mathrm{pHluorin}$ experiments seem to agree well with the three-pool model: synapses contain a releasable pool, displaying fast initial release (likely due to vesicles docked at the active zone) and a slower release afterward (recycling pool). A substantial "reluctant" pool exists - the reserve pool. Exocytosis from this non-releasable pool may be induced through lower frequency stimulation (in what would mean that pHluorins also report the intermixing of the recycling and reserve pools upon physiological stimulation). When stimulating hippocampal neurons continuously at $5 \mathrm{~Hz}$ for $3 \mathrm{~min}$ (900 action potentials) in bafilomycin, fluorescence increased linearly to almost the levels reached by a $30 \mathrm{~Hz}$ stimulation pulse thought to release the entire recycling pool (900 action potentials, Fernandez-Alfonso and Ryan, 2004). It is possible that longer stimulation at low frequency would have resulted in a further increase in fluorescence, recruiting vesicles from the reserve pool, as in the electrophysiology experiments of Ikeda and Bekkers (2009) - an idea which still awaits thorough testing.

\section{"NEW" POOLS: THE CONTROVERSY ON THE SPONTANEOUSLY RELEASING POOL}

Spontaneous vesicle fusion is a phenomenon observed at all synapses. This observation has been fundamental for understanding synaptic transmission, as it forms the basis of quantal neuroscience. At the beginning of the 1950s, while electrophysiologically investigating postsynaptic potentials at frog muscles, Fatt and Katz observed the occurrence of spontaneous small monophasic potentials (with quite constant amplitude, Fatt and Katz, 1952; Boyd and Martin, 1956a). Further analysis revealed that responses to stimulation were made up of single unitary potentials, which were identical in size and shape to the spontaneous potentials (del Castillo and Katz, 1954; Boyd and Martin, 1956b). These units were termed quanta, and were later shown to correspond to the fusion of single vesicles (Katz, 2003).

Although the quantal hypothesis is based on the assumption that single spontaneous quanta are identical to the building blocks of stimulated release, this has never been demonstrated beyond doubt. There is substantial evidence, from many preparations, that basically all vesicles can be released upon stimulation (see above), which would leave few vesicles specialized for spontaneous release. Conversely, prolonged spontaneous release in absence of recycling removes most vesicles in the frog NMJ (Henkel and Betz, 1995; Rizzoli and Betz, 2002). Also, the two types of release are regulated by similar means; for example, both are enhanced upon increased calcium concentration (see Angleson and Betz, 2001; Sara et al., 2005). As mice with defective copies of synaptotagmin I, the major calcium sensor of synchronous neurotransmitter release, did not display any changes in spontaneous release (Geppert et al., 1994), it is highly likely that a different molecule is the calcium sensor for spontaneous release (Groffen et al., 2010) - with the same vesicles perhaps using both sensors. Finally, a further method of stimulation, application of hypertonic solution, releases both the RRP (Rosenmund and Stevens, 1996) and spontaneously recycling vesicles (Kashani et al., 2001).

Direct evidence for an isolated spontaneously recycling pool has been gained recently by studies involving styryl (FM) dyes. In hippocampal cultures, vesicles labeled with the styryl dye FM 2-10 under spontaneous recycling were reluctantly destained (re-released) upon stimulation (Sara et al., 2005). Furthermore, inhibiting the refilling of spontaneously recycling vesicles with neurotransmitter (by applying a vesicular proton pump blocker, folimycin) specifically depleted the spontaneously recycling vesicles, without affecting stimulated release. However, the notion of two distinct vesicle pools driving spontaneous and activity-dependent release was contested shortly afterwards, in similar experiments; using improved imaging/analysis protocols, and relying on the brighter dye FM 1-43, Groemer and Klingauf (2007) demonstrated that stimulation can re-release efficiently both spontaneously labeled and actively labeled vesicles, while application of folimycin during stimulation depleted neurotransmitter release from both actively recycling and spontaneously recycling vesicles (with the latter conclusion also being supported by experiments from an additional investigation, Ikeda and Bekkers, 2009). Thus, although the experiments performed in this study were not precisely identical to those from the first one (Sara et al., 2005), the contradiction was clear and direct.

Recently, this issue has been readdressed (Chung et al., 2010); interestingly, it appeared that FM 2-10, but not FM 1-43, selectively labels spontaneously recycling vesicles. It is difficult to pinpoint how this is achieved. Differences between the hydrophobicities of FM dyes have been used in the past to label different vesicle pools (Richards et al., 2000), although the differences were assigned to FM 2-10, but not FM 1-43, washing from membrane infoldings which gave rise to the reserve vesicles; if enough time was given for the infoldings to bud the reserve vesicles, no wash-off of FM 2-10 could take place, and the pools were labeled identically with the two dyes. As no rapid wash-off appears to take place with spontaneous recycling (where the dye is applied for many minutes), the difference between FM 2-10 and FM 1-43 possibly relies on another parameter (although mechanisms involving wash-off from infoldings, and/or penetration into membrane recycling intermediates could still play a significant role). One other difference between labeling with FM 2-10 and FM 1-43 can be suggested when comparing the concentrations of the dyes used - FM 2-10 is used at $400 \mu \mathrm{M}$, while FM 1-43 is used at or below $10 \mu \mathrm{M}$. With the FM dyes acting as detergent molecules, they perturb vesicle dynamics 
when used in high concentrations, which may thus lead to differences between FM 2-10 and FM 1-43 (Zhu and Stevens, 2008). Finally, another study suggested that FM 1-43 can also be used to differentiate between spontaneously and actively recycling vesicles in brain slices (Mathew et al., 2008) - to some extent further confusing the FM dye issue.

Two further pieces of evidence argue for the presence of separate pools of actively and spontaneously recycling vesicles. First, application of dynasore, an inhibitor of the endocytic GTPase dynamin (Newton et al., 2006) blocked activity-dependent, but not spontaneous release (Chung et al., 2010). Second, a novel labeling technique has been used to test this issue: the vesicular protein synaptobrevin was tagged with a biotin acceptor peptide and was coexpressed with a biotin ligase in cultured hippocampal neurons (Fredj and Burrone, 2009). This procedure resulted in the biotinylation of synaptobrevin, with biotin detected upon exocytosis by fluorescently labeled streptavidin. By employing differently colored streptavidin at rest or under high potassium stimulation, the vesicles involved in both release modes could be selectively stained; the spontaneously and actively recycling vesicles appeared to be completely independent.

In summary, the question whether spontaneous and activitydependent release originate from the same vesicle pool or whether two distinct pools drive these modes of release is still not resolved-far from it. One comment is that the model system used in most of the studies described above, cultured hippocampal neurons obtained from postnatal rats, is highly dependent on culturing/stimulation conditions, with slight differences potentially inducing conflicting results. The choice of cultures of slightly different ages, densities or activity rates may also induce differences. Recently, more "natural" preparations have been used, such as brain slices from animals from postnatal days 6-8 (Chung et al., 2010) or 18-25 (Mathew et al., 2008); this approach should be continued, with more experiments performed on acute slices, and the range of preparations should be expanded to other "classical" models such as the different NMJs.

We conclude that this issue remains controversial, and, since it was mainly tested in only two preparations (hippocampal cultures and brain slices), it is too early to introduce the spontaneously recycling vesicles in the general pool model (Figure 1). Interestingly, the intermixing between the recycling and reserve vesicles may explain some of the conflicting results. Spontaneous labeling experiments are typically longer-lasting than active stimulation experiments, and therefore offer more time for the newly recycled vesicles to mix into the reserve pool, from which they would be harder to re-release (although one should mention that this issue has been at least partially tested, and found not to affect greatly the difference between spontaneously and actively recycling vesicles, Sara et al., 2005).

\section{"NEW" POOLS: THE SURFACE POOL}

The first investigations of vesicular proteins present on the plasma membrane indicated hardly any surface expression of synaptophysin in the frog NMJ (Valtorta et al., 1988) or of synaptotagmin in rat hippocampal neurons (Kraszewski et al., 1995). However, Gandhi and Stevens (2003) used synaptobrevin-pHluorin to visualize the recycling of single vesicles in hippocampal cultures. Occasionally, action potentials were followed by the endocytosis of single quanta of pHluorin, demonstrating the retrieval of "stranded" vesicle membrane upon stimulation. "Stranded" vesicles (vesicles present on the plasma membrane before stimulation) therefore appeared to be an integral part of vesicle recycling.

The stranded molecules organized in clusters of synaptic vesicle protein, which again were clearly involved in synaptic vesicle recycling, as they were endocytosed relatively rapidly (Willig et al., 2006). More direct experiments investigated the issue by using pHluorins and removing the surface-expressed proteins either by illumination-induced bleaching (Fernandez-Alfonso et al., 2006) or by inserting a TEV-protease cleavage site in the construct and removing surface-expressed pHluorin enzymatically (Wienisch and Klingauf, 2006). The non-fluorescent surface molecules were taken up during stimulation, again demonstrating that they can take part in vesicle recycling.

The size of the stranded pool varies somewhat depending upon the protein and the tool used: synaptobrevin-pHluorin has been reported to have a surface expression of 10-15\% (Sankaranarayanan et al., 2000) or up to $\sim 24 \%$ (Granseth et al., 2006); $~ 8 \%$ has been reported for synaptophysin (Granseth et al., 2006); 2\% for the glutamate transporter vGlut1 (Balaji and Ryan, 2007); and $24 \%$ for synaptotagmin (Wienisch and Klingauf, 2006). Finally, studying native synaptotagmin localization reported that $\sim 19 \%$ of all synaptotagmin is present on the surface (Opazo et al., 2010) - with all values obtained from hippocampal cultured neurons. Undoubtedly, some of the differences may be due to overexpression artifacts especially as the pHluorins do not appear to be organized in clusters on the plasma membrane, while the native synaptic proteins are always found clustered (Opazo et al., 2010), in what would appear to be a tag-induced mislocalization.

At least for synaptotagmin, the surface pool of the native protein was identical in size with the RRP (Opazo et al., 2010). By definition, the surface pool cannot be regarded as part of the RRP, but of the recycling pool - as these are certainly rapidly recycling vesicles, although not "ready for release", but rather "ready for endocytosis" (Figure 1C). However, it would be interesting to know whether endocytosed surface pool vesicles display different, possibly higher release properties than the remaining recycling pool vesicles, underlining their interplay with the RRP.

\section{"NEW" POOLS: THE SUPER-POOL}

Since the start of synaptic research, it has been generally assumed that synapses function as individual units, with individual vesicle recycling and little intermixing between terminals. The concept is in perfect agreement with results obtained from cultured hippocampal neurons, indicating that after stimulation synaptobrevin is retrieved from the presynaptic membrane and from adjacent axonal regions by endocytosis, and is then returned to the vesicle cluster (Li and Murthy, 2001). Synapsin, a protein thought to participate in interlinking synaptic vesicles, similarly diffuses from the synapse into the axon during activity, and returns upon cessation of stimulation (Chi et al., 2001).

However, recent studies have shown that the exchange of vesicles among boutons is actually substantial: vesicles (and even whole release-competent vesicle clusters) labeled with styryl dyes in cultured hippocampal neurons were transported along axons both antero- and retrogradely (Krueger et al., 2003; Darcy et al., 2006). 
FRAP (fluorescence recovery after photobleaching), a method involving the bleaching of a small part of the preparation followed by monitoring the fluorescence recovery in this region, demonstrated the entry of a substantial proportion of vesicles into synaptic boutons, coming from neighboring boutons. With the advent of super-resolution microscopy, the exchange between synapses could not only be confirmed, but also monitored on a single vesicle level (Westphal et al., 2008; Kamin et al., 2010). Recently, the concept of the super-pool was also extended to hippocampal slices (Staras et al., 2010).

As exchanged vesicles could undergo fusion with normal kinetics (Darcy et al., 2006), the super-pool seems to overlap at least partially with the recycling pool. However, reserve pool vesicles are exchanged as well (Darcy et al., 2006; Kamin et al., 2010), and synaptobrevin-pHluorin molecules from both the recycling and the reserve pool vesicles were found to be exchanged between synapses (Fernandez-Alfonso and Ryan, 2008). Thus, the super-pool extends the general three-pool model: the pools defined in the past are no longer limited to single synaptic boutons, but instead can be exchanged across multiple synapses (Figure 1C).

\section{VESICLE MOBILITY}

A general assumption in synaptic vesicle recycling has been that synaptic vesicles are quite stable at rest, but move actively toward active zones upon stimulation. That most vesicles are stable at rest has been repeatedly verified, with movement of styryl dye labeled vesicles clearly constrained in the frog NMJ (Betz and Bewick, 1992) or in hippocampal cultures (Jordan et al., 2005; Shtrahman et al., 2005). Vesicle mobility could only be observed under non-physiological conditions, such as the treatment with the phosphatase inhibitor okadaic acid (Betz and Henkel, 1994; Shtrahman et al., 2005). Vesicles did not appear to move much more upon stimulation (Betz et al., 1992), with their mobility remaining limited (Henkel et al., 1996; Lemke and Klingauf, 2005) - contradicting the old expectation that vesicles should move to the active zone.

A more recent FRAP study reported that vesicles in the recycling pool of the frog NMJ were mobile at rest, while the larger reserve pool was immobile (Gaffield et al., 2006). The mobility of the recycling vesicles never increased under stimulation, but the reserve pool became mobile after prolonged heavy stimulation, eventually reaching the mobility of the recycling pool (Gaffield et al., 2006). A similar study in the mouse NMJ noted that the vesicles appeared to be immobile at room temperature, but that their mobility increased strongly at physiological temperature (Gaffield and Betz, 2007); with most studies performed at room temperature, this study still awaits confirmation in other mammalian preparations. However, a simple conclusion can be already gathered: synaptic vesicles do not travel toward the active zone upon stimulation. Rather, the majority (reserve pool) may be kept "fixed", with only the recycling pool moving (randomly?) both at rest and under stimulation.

To obtain a better understanding of vesicle mobility, single synaptic vesicles have been recently tracked by stimulated emission depletion (STED) microscopy (Westphal et al., 2008). Conventional light microscopy is well-suited for live imaging, but is limited by the diffraction limit of light (Abbe, 1873): the distance that can be resolved between two objects is inversely proportional to the light's angular distribution, and directly proportional to the wavelength. For over a century, this diffraction limit (which translates into at least $\sim 200 \mathrm{~nm}$ for most light microscopy applications) could not be overcome. STED microscopy is one of several new imaging concepts (Hell, 2007) which break the diffraction barrier. In STED, the diffraction-limited excitation beam is overlaid with a doughnutshaped depletion beam (Hell and Wichmann, 1994). This depletion beam deexcites (depletes) the fluorescent molecules by stimulated emission. As the depletion beam has nearly zero intensity in the center, the fluorophores excited in this very small central spot are not quenched, and thus fluorescence is collected only from a small sub-diffraction spot. Using this method, substantial vesicle movement was detected in cultured hippocampal neurons (Westphal et al., 2008); vesicles typically moved randomly, although directional movement was also observed, especially in axonal regions. Intriguingly, vesicles were occasionally trapped in "hot spots", areas of low mobility which assembled in what resembled synaptic boutons, possibly reflecting "pockets" within vesicle clusters. These observations correspond to the so-called "stick-and-diffuse" model of vesicle movement, in which vesicles diffuse freely and bind from time to time to structures within the terminal, becoming transiently stuck (Shtrahman et al., 2005; Yeung et al., 2007). These findings were extended in a subsequent study (Kamin et al., 2010), which also added the observation that whereas recently endocytosed vesicles were quite mobile, they lost this mobility over hours, as they integrated into the stable vesicle cluster. Importantly, both recently endocytosed and "mature" cluster-integrated vesicles did not display any change in mobility upon stimulation, in agreement with the studies mentioned above. However, when activity was inhibited by blocking action potentials via tetrodotoxin, the movement of the vesicles decreased significantly faster (within a few minutes).

With recently endocytosed vesicles being undoubtedly part of the recycling pool, and with the "mature" ones likely representing the reserve pool (Kamin et al., 2010), it is again tempting to hypothesize that the recycling pool is mobile, while the reserve is not (as in the frog, see above). The fact that the mobile vesicles became fixed over hours is in good agreement with a high intermixing rate of reserve and recycling vesicles, as in the pool model we proposed above (Figure 1C).

In the model of synaptic pools we propose (Figure 1C), we termed the reserve pool "mature". This terminology refers to the fate of a vesicle after recycling, considering endocytosis to be the first event in the life of the particular vesicle. A newly endocytosed vesicle belongs to the mobile recycling pool (Kamin et al., 2010), and therefore is more likely to release again (and maintain its recycling pool tag). Over time, these vesicles lose both their mobility and their ability to release, and integrate into clusters. From a temporal point of view, they are "older" vesicles, with "mature" being the simplest term to define them. This phenomenon has been substantiated not only by STED imaging of vesicles (Kamin et al., 2010), but also by several FM dye release studies (for example Richards et al., 2000, 2003; Pyle et al., 2000; Rizzoli and Betz, 2004). From such studies it is clear that newly recycled vesicles turn over time into "mature", less releasable vesicles - many vesicles become reluctantly releasable, or occasionally cannot even be released by massive activity such as thousands of stimuli at high frequency. 
Clearly, some reserve pool vesicles would eventually need to replace the newly cluster-integrated recycling vesicles (or the synapse would run out of releasable vesicles). However, it has been strangely difficult to observe this by FM imaging - slowly releasable vesicles (reserve vesicles) remain so over time. A simple interpretation is that they do indeed turn into mobile recycling vesicles, but only after fusion: after releasing their contents of FM dye, and becoming invisible for fluorescence microscopy. In agreement with this hypothesis, these vesicles appear immobile over tens of minutes under low- or moderate-frequency stimulation (although the dye is slowly lost from the terminals, indicating that the vesicles do fuse to release it, without becoming more mobile for any substantial time period before the release step, Betz and Henkel, 1994; see also Henkel et al., 1996; Gaffield et al., 2006). In contrast, when using antibody labeling, rather than FM dyes, the mobility of (previously immobile) reserve vesicles increases dramatically after release and endocytosis (their mobility can be still followed as these vesicles remain labeled after the endo-exocytosis step - unlike FM dyes, the antibodies are not lost upon fusion). This experiment (Kraszewski et al., 1996) thus provides a good example for reserve vesicles becoming mobile recycling vesicles after endocytosis. How would largely immobile vesicles reach the active zones to fuse (and then become mobile)? As indicated by Kamin et al. (2010), the exchange of vesicles between synapses is equally efficient for reserve or recycling vesicles, despite the differences in their mobility (see also Darcy et al., 2006; FernandezAlfonso and Ryan, 2008). Therefore, it is not inconceivable that the largely immobile reserve vesicles are transported occasionally to the active zone, in order to be able to dock and fuse. In agreement with this hypothesis, substantial numbers of reserve vesicles are docked at active zones (Rizzoli and Betz, 2004; Denker et al., 2009; see above).

Thus, we do not see reserve vesicles as "immature" vesicles which would slowly mature into recycling ones. The reserve status represents the eventual fate for all recycling vesicles, being thus the last step in the endocytotic cycle of the recycling vesicle. We therefore decided to use the term "maturation" for the transformation of the mobile recycling vesicle into the fixed reserve vesicle.

What would restrict the movement of reserve vesicles, and why would this restriction apply less to the recycling pool? Synapsin has been proposed to interlink vesicles, especially the reserve pool (Pieribone et al., 1995), and synapsin knockout mice typically have relatively serious synaptic phenotypes, such as higher synaptic depression, lower vesicle numbers or difficulties in recovering after stimulation (for example Li et al., 1995; Gitler et al., 2004). However, elimination of synapsin does not remove all filaments interlinking vesicles (Siksou et al., 2007), and the synaptic vesicle mobility in synapsin-null mice was indistinguishable from that of wild type mice (Gaffield and Betz, 2007). Nevertheless, synapsin would still make a perfect candidate for the "glue" of the reserve pool: it does bind vesicles, but not fused vesicles (Bloom et al., 2003), and therefore vesicles which frequently recycle may bind fewer synapsin molecules, and as a consequence remain mobile (as will be further discussed below). In agreement with this model, the ribbon synapses of the organ of Corti and the retina, which lack synapsin (Mandell et al., 1990), display high vesicle mobility (Holt et al., 2004).
At any rate, a conclusion on the general vesicle mobility we propose is that a certain amount of mobility of the recycling pool is sufficient both at rest and under normal (physiological) stimulation conditions. Therefore, no major changes in vesicle mobility should be expected upon (stimulated) activity.

\section{MODELS OF VESICLE RECYCLING}

One of the long-standing debates in the synaptic field is whether vesicles exocytose by collapsing into the plasma membrane, to be later retrieved by clathrin-coat mediated endocytosis, or whether they only fuse transiently, through a small fusion pore (kiss-andrun). This question has been focused on for more than three decades (see for example the classical review of Ceccarelli and Hurlbut, 1980). Whereas the existence of clathrin-mediated endocytosis at synapses is well-established (and discussed in several excellent recent reviews, for example Granseth et al., 2007; Jung and Haucke, 2007; Maritzen et al., 2010; Pechstein et al., 2010; Royle and Lagnado, 2010), the role of the kiss-and-run pathway is still controversial. Furthermore, it is unclear whether different vesicle pools use different modes of recycling.

Several years ago, two important pieces of evidence were obtained in cultured hippocampal neurons. The fusion of single synaptic vesicles was imaged using pHluorins (Gandhi and Stevens, 2003) and FM dyes (Aravanis et al., 2003), and both studies concluded that synaptic vesicles can fuse via kiss-and-run: rapidly endocytosing vesicles were observed in pHluorin imaging, and FM dye release suggested that the same individual vesicle can release multiple times, without losing the full amount of FM dye - which would have been lost upon collapsing into the membrane. In agreement with these studies, a substantial amount of FM dye is retained in recycling vesicles (Harata et al., 2006).

However, a subsequent study of FM dye release indicated that at least a large fraction of the vesicles participate in full fusion, losing their entire FM dye contents (Chen et al., 2008), and another study, using pHluorins, suggested that inhibition of clathrin activity removes essentially all endocytosis (Granseth et al., 2006), arguing in favor of full collapse followed by clathrin-mediated endocytosis as the major mechanism of vesicle recycling. Full collapse is also in agreement with the possibility to label recycling vesicles by bulky molecules such as antibodies (Willig et al., 2006).

New evidence for kiss-and-run was obtained from a study taking advantage of the size and $\mathrm{pH}$-dependent fluorescence change of quantum dots (Zhang et al., 2009). Vesicles were labeled by endocytosis of quantum dots; upon subsequent fusion, the quantum dots displayed increased fluorescence, due to the change from acidic to neutral $\mathrm{pH}$, as for the pHluorins (see above), without, however, leaving the vesicle they occupied. The study also suggested that the same synaptic vesicles may participate in both kissand-run and full collapse fusion - which explains why inhibition of one of the pathways may eventually remove all endocytosis (see above; however, see also Granseth et al., 2009, for alternative interpretations).

A further prominent argument for kiss-and-run fusion has been derived from capacitance measurements on the calyx of Held, which indicated very rapid endocytosis (Sun et al., 2002; Wu et al., 2005). Capacitance measurements provide the advantage of much higher time resolution, as compared to imaging. However, 
using the same technique and an inhibitor of fusion (botulinum toxin), it was suggested that at least some of the fast capacitance transients were not related to synaptic transmission (Yamashita et al., 2005). More recent evidence investigating the fusion and endocytosis of single vesicles on small membrane areas has again provided arguments in favor of rapid endocytosis (He et al., 2006), which, intriguingly, may not actually work for the RRP, as has been often assumed, but for other (presumably recycling pool) vesicles ( $\mathrm{Wu}$ and $\mathrm{Wu}, 2009$ ). One may tentatively conclude that, while the debate on the role of kiss-and-run still continues, it is not inconceivable that the same vesicles could participate in both kiss-and-run and full fusion, perhaps at different times or under different conditions - although the molecular mechanisms behind kiss-and-run fusion are still to be identified. An interesting related finding has been recently reported in cultured hippocampal neurons: single vesicle fusion followed by dual retrieval (Zhu et al., 2009). The authors employed a new pHluorin variant, in which multiple pHluorins are tagged to synaptophysin, providing a better signal-to-background ratio. The reporter allowed for tracking of individual vesicles following exocytosis, and revealed that the release of one vesicle was followed by two internalization events, one with a rapid and one with a slow time-course. Although this paradigm appears to be in agreement only with full fusion (in kiss-and-run one vesicle could not separate into two), it points to a heterogeneity in vesicle retrieval which has been rarely hypothesized in the past.

Two other recycling models have been debated, with most studies concurring on their existence: bulk endocytosis, in which vesicles are generated from large membrane infoldings arising from the exocytosis of many vesicles in quick succession, and endosomal sorting, where the recently endocytosed vesicles fuse with a sorting endosome, from which new vesicles will bud in turn. Substantial evidence for bulk endocytosis has been obtained, for example, in cultured cerebellar granule neurons (Clayton et al., 2008) and the calyx of Held (Wu and Wu, 2007). Importantly, one of the molecular players involved in bulk endocytosis was recently identified in the lamprey, syndapin (Andersson et al., 2008). Although already proposed by Heuser and Reese (1973), endosomal sorting has received relatively limited evidence. In an important study, endosomes were labeled in the Drosophila larva NMJ, and they were observed to recycle membrane along with the synaptic vesicles one of the only studies to date to show the recycling of endosomal membrane upon stimulation in synapses (Wucherpfennig et al., 2003). More recent findings from hippocampal cultures include changes in synaptic vesicle recycling upon inhibition or knockout of members of endosomal pathways (Voglmaier et al., 2006; Glyvuk et al., 2010). In addition, recently endocytosed vesicles can fuse with purified bona fide early endosomes (Rizzoli et al., 2006), although only to a rather limited level. Interestingly, the large organelles associated with bulk (or endosomal) endocytosis may depend upon the integrity of the active zones. Substantial endocytosis close to active zones has been observed in the past, occasionally even in the form of bulk-like infoldings (for example in the frog NMJ, Ceccarelli et al., 1979; Drosophila photoreceptor cells, Koenig and Ikeda, 1996; the Drosophila NMJ, Koenig et al., 1998; or the snake NMJ, Teng et al., 1999; Teng and Wilkinson, 2000), and large membrane compartments (possibly arising from bulk endocytosis) replaced much of the vesicle content of hair cells (which participate in synaptic transmission in the auditory pathway) when active zones were perturbed (Khimich et al., 2005).

We would tentatively conclude that multiple vesicle recycling routes are used by synaptic vesicles, with the choice of pathway possibly depending on stimulation frequency - with kiss-and-run, for example, appearing to increase upon rapid stimulation (Zhang et al., 2009). For instance, bulk endocytosis seems to be associated specifically with strong stimulation protocols, which may selectively trigger release of reserve pool vesicles; however, the specific use of any one of the other pathways (kiss-and-run, clathrin-mediated endocytosis, endosomal sorting) by specific pools of vesicles remains to be elucidated.

\section{MOLECULAR TAGS DISTINGUISHING BETWEEN THE POOLS?}

Synaptic vesicle pools may be differentiated by a molecular tag, by a protein (or set of proteins) which is present selectively on one pool of vesicles. The complement of proteins associated with synaptic vesicles has been recently clarified (Takamori et al., 2006). Some proteins, such as synaptobrevin, synaptotagmin or synaptophysin are present at high levels (about 70 copies of synaptobrevin would be present on one average vesicle, with $\sim 15$ for synaptotagmin I and $\sim 30$ for synaptophysin). These abundant proteins are unlikely to represent pool tags, but rather a basic protein set common to all vesicles. Other proteins, however, were much less abundant such as the endosomal SNARE fusion proteins; these are present in one or a few copies per vesicle (Takamori et al., 2006). It is easy to hypothesize that these unabundant proteins are actually present in high copy numbers only on some vesicles (in a specific pool of vesicles), perhaps thus representing pool tags.

The intermixing between vesicle pools, as observed in many preparations on timescales of minutes or at most hours, could perhaps arise from the intermixing of synaptic vesicle components during recycling. With one vesicle separating in two components upon fusion (Zhu et al., 2009), the different pool types may exchange biochemical tags on the plasma membrane. However, recent evidence argues against a strong intermixing of vesicle components upon fusion (Opazo et al., 2010). Furthermore, intermixing after exocytosis would require that both recycling and reserve pool components are always present on the plasma membrane, which would suggest that both pools recycle simultaneously - in disagreement with most vesicle pool studies.

However, as indicated above, it is also possible that the pool tag is not a permanent one - i.e., not a transmembrane protein which would only be lost from the vesicles upon recycling. Rather, it is possible that pool status is determined by affiliation with a soluble protein. As suggested above, synapsin binds normal, internalized vesicles, but not fused ones (Bloom et al., 2003). Upon endocytosis, the recycling vesicles would remain mobile as long as they are not bound by a sufficient number of synapsin molecules (with the average vesicle containing $\sim 8$ synapsin molecules, Takamori et al., 2006). Upon strong synapsin binding (Bloom et al., 2003), the recycling pool vesicles would be cross-linked to other vesicles within the cluster, and would turn into (immobile) reserve pool vesicles. Synapsin binding might well be regulated by activity, as inactivity promotes loss of mobility (see above, Kamin et al., 2010) - although clear evidence for such a model is yet to be obtained. 


\section{CONCLUSION}

The synaptic vesicle pool field has strongly advanced in the last few years, with some new concepts, such as the surface pool or the superpool, being now well accepted. Others are still controversial, such as the role and pool affiliation of the spontaneously fusing vesicles. However, the field of vesicle pool research seems to have approached a stage at which further understanding depends not only on technical improvements, but also on a shift towards more physiologically relevant studies - observing not what the system can be forced to do under strong stimulation, but how it works under native conditions. In this context, the role of the reserve pool needs further clarification-are these vesicles ever called into action in vivo? In other words, is activity in vivo strong enough to require the use of reserve vesicles?

A further major challenge for the pool field will be the in vivo application of imaging approaches that were established mainly in vitro. The development and use of pHluorin tools in mice, for

\section{REFERENCES}

Abbe, E. (1873). Beiträge zur Theorie des Mikroskops und der mikroskopischen Wahrnehmung. Arch. Mikros. Anat. 9, 55.

Akbergenova, Y., and Bykhovskaia, M. (2007). Synapsin maintains the reserve vesicle pool and spatial segregation of the recycling pool in Drosophila presynaptic boutons. Brain Res. 1178, 52-64.

Akbergenova, Y., and Bykhovskaia, M. (2009). Stimulation-induced formation of the reserve pool of vesicles in Drosophila motor boutons. J. Neurophysiol. 101, 2423-2433.

Andersson, F., Jakobsson, J., Low, P., Shupliakov, O., and Brodin, L. (2008). Perturbation of syndapin/PACSIN impairs synaptic vesicle recycling evoked by intense stimulation. J. Neurosci. 28, 3925-3933.

Angleson, J. K., and Betz, W. J. (2001). Intraterminal $\mathrm{Ca}(2+)$ and spontaneous transmitter release at the frog neuromuscular junction. J. Neurophysiol. 85, 287-294.

Aravanis, A. M., Pyle, J. L., and Tsien, R.W. (2003). Single synaptic vesicles fusing transiently and successively without loss of identity. Nature 423, 643-647.

Balaji, J., and Ryan, T. A. (2007). Singlevesicle imaging reveals that synaptic vesicle exocytosis and endocytosis are coupled by a single stochastic mode. Proc. Natl. Acad. Sci. U.S.A. 104, 20576-20581.

Betz, W. J., and Bewick, G. S. (1992). Optical analysis of synaptic vesicle recycling at the frog neuromuscular junction. Science 255, 200-203.

Betz, W. J., Bewick, G. S., and Ridge, R. M. (1992). Intracellular movements of fluorescently labelled synaptic vesicles in frog motor nerve terminals during nerve stimulation. Neuron 9 , 805-813.
Betz, W. J., and Henkel, A. W. (1994). Okadaic acid disrupts clusters of synminals. J. Cell Biol. 124, 843-854.

Birks, R., and MacIntosh, F. C. (1961). Acetylcholine metabolism of a sympathetic ganglion. Can. J. Biochem. Physiol. 39, 787-827.

Bloom, O., Evergren, E., Tomilin, N., Kjaerulff, O., Low, P., Brodin, L., Pieribone, V. A., Greengard, P., and Shupliakov, O. (2003). Colocalization of synapsin and actin during synaptic vesicle recycling. J. Cell Biol. 161, 737-747.

Bollmann, J. H., Sakmann, B., and Borst, J. G. G. (2000). Calcium sensitivity of glutamate release in a calyx-type terminal. Science 289, 953-957.

Boyd, I. A., and Martin, A. R. (1956a). Spontaneous subthreshold activity at mammalian neural muscular junctions. J. Physiol. 132, 61-73.

Boyd, I. A., and Martin, A. R. (1956b). The end-plate potential in mammalian muscle. J. Physiol. 132, 74-91.

Ceccarelli, B., Grohovaz, F., and Hurlbut, W. P. (1979). Freeze-fracture studies of frog neuromuscular junctions during intense release of neurotransmitter. II. Effects of electrical stimulation and high potassium. J. Cell. Biol. 81, 178-192.

Ceccarelli, B., and Hurlbut, W. P. (1980). Vesicle hypothesis of the release of quanta of acetylcholine. Physiol. Rev. 60, 396-441.

Ceccarelli, B., Hurlbut, W. P., and Mauro, A. (1972). Depletion of vesicles from frog neuromuscular junctions by prolonged tetanic stimulation. J. Cell Biol. $54,30-38$.

Chen, X., Barg, S., and Almers, W. (2008). Release of the styryl dyes from single synaptic vesicles in hippocampal neurons. J. Neurosci. 28, 1894-1903. aptic vesicles in frog motor nerve ter-

investigation of activity in both excised NMJs (Tabares et al., 2007; Wyatt and Balice-Gordon, 2008) and entire brain regions (Li et al., 2005; Petzold et al., 2008), is clearly an important step forward.

\section{ACKNOWLEDGMENTS}

Annette Denker is a doctoral student of the Ph.D. program "Molecular Biology" - International Max Planck Research School and the Göttingen Graduate School for Neurosciences and Molecular Biosciences (GGNB) (DFG grant GSC 226/1) at the Georg August University Göttingen. Annette Denker is supported by a Boehringer Ingelheim Fonds PhD Fellowship. Silvio O. Rizzoli acknowledges the support of a Starting Grant from the European Research Council, Program FP7, and of the DFG Research Center for Molecular Physiology of the Brain (CMPB)/ Excellence Cluster 171.

Chi, P., Greengard, P., and Ryan, T. A. (2001). Synapsin dispersion and reclustering during synaptic activity. Nat. Neurosci. 4, 1187-1193.

Chung, C., Barylko, B., Leitz, J., Liu, X., and Kavalali, E. T. (2010). Acute dynamin inhibition dissects synaptic vesicle recycling pathways that drive spontaneous and evoked neurotransmission. J. Neurosci. 30, 1363-1376.

Clayton, E. L., Evans, G. J., and Cousin, M. A. (2008). Bulk synaptic vesicle endocytosis is rapidly triggered during strong stimulation. J. Neurosci. 28, 6627-6632.

Darcy, K. J., Staras, K., Collinson, L. M. and Goda, Y. (2006). Constitutive sharing of recycling synaptic vesicles between presynaptic boutons. Nat. Neurosci. 9, 315-321.

de Lange, R. P., de Roos, A. D., and Borst, J. G. (2003). Two modes of vesicle recycling in the rat calyx of Held. J. Neurosci. 23, 10164-10173.

del Castillo, J., and Katz, B. (1954). Quantal components of the end-plate potential. J. Physiol. 124, 560-573.

Delgado, R., Maureira, C., Oliva, C. Kidokoro, Y., and Labarca, P. (2000). Size of vesicle pools, rates of mobilization, and recycling at neuromuscular synapses of a Drosophila mutant, shibire. Neuron 28, 941-953.

Denker, A., Krohnert, K., and Rizzoli, S. O. (2009). Revisiting synaptic vesicle pool localization in the Drosophila neuromuscular junction. J. Physiol. 587, 2919-2926.

Elmqvist, D., and Quastel, D. M. (1965). A quantitative study of end-plate potentials in isolated human muscle. J. Physiol. 178, 505-529.

Fatt, P., and Katz, B. (1952). Spontaneous subthreshold activity at motor nerve endings. J. Physiol. 117, 109-128.

Fernandez-Alfonso, T., Kwan, R., and Ryan, T. A. (2006). Synaptic vesicles interchange their membrane proteins with a large surface reservoir during recycling. Neuron 51, 179-186.

Fernandez-Alfonso, T., and Ryan, T. A. (2004). The kinetics of synaptic vesicle pool depletion at CNS synaptic terminals. Neuron 41, 943-953.

Fernandez-Alfonso, T., and Ryan, T. A. (2008).A heterogeneous "resting" pool of synaptic vesicles that is dynamically interchanged across boutons in mammalian CNS synapses. Brain Cell Biol. 36, 87-100.

Fredj, N. B., and Burrone, J. (2009). A resting pool of vesicles is responsible for spontaneous vesicle fusion at the synapse. Nat. Neurosci. 12, 751-758.

Gaffield, M. A., and Betz, W. J. (2007). Synaptic vesicle mobility in mouse motor nerve terminals with and without synapsin. J. Neurosci. 27, 13691-13700.

Gaffield, M. A., Rizzoli, S. O., and Betz, W. J. (2006). Mobility of synaptic vesicles in different pools in resting and stimulated frog motor nerve terminals. Neuron 51, 317-325.

Gandhi, S. P., and Stevens, C. F. (2003). Three modes of synaptic vesicular recycling revealed by single-vesicle imaging. Nature 423, 607-613.

Geppert, M., Goda, Y., Hammer, R. E., Li, C., Rosahl, T. W., Stevens, C. F., and Sudhof, T. C. (1994). Synaptotagmin I: a major $\mathrm{Ca} 2+$ sensor for transmitter release at a central synapse. Cell 79, 717-727.

Gitler, D., Takagishi, Y., Feng, J., Ren, Y., Rodriguiz, R. M., Wetsel, W. C., Greengard, P., and Augustine, G. J. (2004). Different presynaptic roles of synapsins at excitatory and inhibitory synapses. J. Neurosci. 24, 11368-11380.

Glyvuk, N., Tsytsyura, Y., Geumann, C., D’Hooge, R., Hüve, J., Kratzke, M., Baltes, J., Böning, D., Klingauf, J., 
and Schu, P. (2010). AP-1/sigma1Badaptin mediates endosomal synaptic vesicle recycling, learning and memory. EMBO J. 29, 1318-1330.

Granseth, B., Odermatt, B., Royle, S. J., and Lagnado, L. (2006). Clathrin-mediated endocytosis is the dominant mechanism of vesicle retrieval at hippocampal synapses. Neuron 51, 773-786.

Granseth, B., Odermatt, B., Royle, S. J., and Lagnado, L. (2007). Clathrin-mediated endocytosis: the physiological mechanism of vesicle retrieval at hippocampal synapses. J. Physiol. 585, 681-686.

Granseth, B., Odermatt, B., Royle, S. J., and Lagnado, L. (2009). Comment on "The dynamic control of kiss-and-run and vesicular reuse probed with single nanoparticles". Science 325, 1499; author reply 1499.

Groemer, T. W., and Klingauf, J. (2007). Synaptic vesicles recycling spontaneously and during activity belong to the same vesicle pool. Nat. Neurosci. $10,145-147$.

Groffen, A. J., Martens, S., Diez Arazola, R., Cornelisse, L. N., Lozovaya, N., de Jong, A. P., Goriounova, N. A., Habets, R. L., Takai, Y., Borst, J. G., Brose, N., McMahon, H. T., and Verhage, M. (2010). Doc2b is a highaffinity $\mathrm{Ca} 2+$ sensor for spontaneous neurotransmitter release. Science 327, 1614-1618.

Guatimosim, C., and von Gersdorff, H. (2002). Optical monitoring of synaptic vesicle trafficking in ribbon synapses. Neurochem. Int. 41, 307-312.

Harata, N., Pyle, J. L., Aravanis, A. M., Mozhayeva, M., Kavalali, E. T., and Tsien, R. W. (2001a). Limited numbers of recycling vesicles in small CNS nerve terminals: implications for neural signaling and vesicular cycling. Trends Neurosci. 24, 637-643.

Harata, N., Ryan, T. A., Smith, S. J., Buchanan, J., and Tsien, R. W. (2001b). Visualizing recycling synaptic vesicles in hippocampal neurons by FM 1-43 photoconversion. Proc. Natl. Acad. Sci. U.S.A. 98, 12748-12753.

Harata, N.C., Choi, S., Pyle, J.L., Aravanis, A. M., and Tsien, R. W. (2006). Frequency-dependent kinetics and prevalence of kiss-and-run and reuse at hippocampal synapses studied with novel quenching methods. Neuron 49 , 243-256.

Hayashi, M., Raimondi, A., O'Toole, E., Paradise, S., Collesi, C., Cremona, O., Ferguson, S. M., and De Camilli, P. (2008).Cell- and stimulus-dependent heterogeneity of synaptic vesicle endocytic recycling mechanisms revealed by studies of dynamin 1-null neurons. Proc. Natl. Acad. Sci. U.S.A. 105, 2175-2180.

He, L., Wu, X. S., Mohan, R., and Wu, L. G. (2006). Two modes of fusion pore opening revealed by cell-attached recordings at a synapse. Nature 444 , 102-105.

Hell, S. W. (2007). Far-field optical nanoscopy. Science 316, 1153-1158.

Hell, S. W., and Wichmann, J. (1994). Breaking the diffraction resolution limit by stimulated emission: stimulated-emission-depletion fluorescence microscopy. Opt. Lett. 19, 780-782.

Henkel, A. W., and Betz, W. J. (1995). Monitoring of black widow spider venom (BWSV) induced exo- and endocytosis in living frog motor nerve terminals with FM1-43. Neuropharmacology 34, 1397-1406.

Henkel, A. W., Simpson, L. L., Ridge, R. M., and Betz, W. J. (1996). Synaptic vesicle movements monitored by fluorescence recovery after photobleaching in nerve terminals stained with FM1-43. J. Neurosci. 16, 3960-3967.

Hermann, J., Grothe, B., and Klug, A. (2009). Modeling short-term synaptic plasticity at he calyx of Held using in vivo-like stimulation patterns. $J$. Neurophysiol. 101, 20-30.

Hermann, J., Pecka, M., von Gersdorff, H., Grothe, B., and Klug, A. (2007) Synaptic transmission at the calyx of Held under in vivo-like activity levels. J. Neurophysiol. 98, 807-820.

Heuser, J. E., and Reese, T. S. (1973). Evidence for recycling of synaptic vesicle membrane during transmitter release at the frog neuromuscular junction. J. Cell Biol. 57, 315-344.

Holt, M., Cooke, A., Neef, A., and Lagnado, L. (2004). High mobility of vesicles supports continuous exocytosis at a ribbon synapse. Curr. Biol. $14,173-183$.

Ikeda, K., and Bekkers, J. M. (2009). Counting the number of releasable synaptic vesicles in a presynaptic terminal. Proc. Natl. Acad. Sci. U.S.A. 106, 2945-2950.

Jordan, R., Lemke, E. A., and Klingauf, J. (2005).Visualization of synaptic vesicle movement in intact synaptic boutons using fluorescence fluctuation spectroscopy. Biophys. J. 89, 2091-2102.

Jung, N., and Haucke, V. (2007). Clathrinmediated endocytosis at synapses. Traffic 8, 1129-1136.

Kamin, D., Lauterbach, M. A., Westphal, V., Keller, J., Schönle, A., Hell, S. W., and Rizzoli, S. O. (2010). High- and low-mobility stages in the synaptic vesicle cycle. Biophys. J. 99, 675-684.

Kashani, A. H., Chen, B. M., and Grinnell, A.D. (2001).Hypertonic enhancement of transmitter release from frog motor nerve terminals: $\mathrm{Ca} 2+$ independence and role of integrins. J. Physiol. 530, 243-252.

Katz, B. (2003). Neural transmitter release: from quantal secretion to exocytosis and beyond. J. Neurocytol. $32,437-446$.

Khimich, D., Nouvian, R., Pujol, R., Tom Dieck, S., Egner, A., Gundelfinger, E. D., and Moser, T. (2005). Hair cell synaptic ribbons are essential for synchronous auditory signalling. Nature 434, 889-894.

Koenig, J. H., and Ikeda, K. (1989). Disappearance and reformation of synaptic vesicle membrane upon transmitter release observed under reversible blockage of membrane retrieval. J. Neurosci. 9, 3844-3860.

Koenig, J. H., and Ikeda, K. (1996) Synaptic vesicles have two distinct recycling pathways. J. Cell Biol. 135 , 797-808.

Koenig, J. H., Saito, K., and Ikeda, K. (1983). Reversible control of synaptic transmission in a single gene mutant of Drosophila melanogaster. J. Cell Biol. 96, 1517-1522.

Koenig, J. H., Yamaoka, K., and Ikeda, K. (1998). Omega images at the active zone may be endocytotic rather than exocytotic: implications for the vesicle hypothesis of transmitter release. Proc. Natl. Acad. Sci. U.S.A. 95 , 12677-12682.

Kraszewski, K., Daniell, L., Mundigl, O., and De Camilli, P. (1996). Mobility of synaptic vesicles in nerve endings monitored by recovery from photobleaching of synaptic vesicleassociated fluorescence. J. Neurosci. 16, 5905-5913.

Kraszewski, K., Mundigl, O., Daniell, L., Verderio, C., Matteoli, M., and De Camilli, P. (1995). Synaptic vesicle dynamics in living cultured hippocampal neurons visualized with CY3conjugated antibodies directed against the lumenal domain of synaptotagmin. J. Neurosci. 15, 4328-4342.

Krueger, S. R., Kolar, A., and Fitzsimonds, R. M. (2003). The presynaptic release apparatus is functional in the absence of dendritic contact and highly mobile within isolated axons. Neuron 40 945-957.

Kuromi, H., and Kidokoro, Y. (1998). Two distinct pools of synaptic vesicles in single presynaptic boutons in a temperature-sensitive Drosophila mutant, shibire. Neuron 20, 917-925.

Kuromi, H., and Kidokoro, Y. (1999). The optically determined size of exo/endo cycling vesicle pool correlates with the quantal content at the neuromuscular junction of Drosophila larvae. $J$. Neurosci. 19, 1557-1565.

Kuromi, H., and Kidokoro, Y. (2000). Tetanic stimulation recruits vesicles from reserve pool via a cAMPmediated process in Drosophila synapses. Neuron 27, 133-143.

Kuromi, H., and Kidokoro, Y. (2002). Selective replenishment of two vesicle pools depends on the source of $\mathrm{Ca} 2+$ at the Drosophila synapse. Neuron 35 , 333-343.

Lemke, E. A., and Klingauf, J. (2005). Single synaptic vesicle tracking in individual hippocampal boutons at rest and during synaptic activity. $J$. Neurosci. 25, 11034-11044.

Li, L., Chin, L. S., Shupliakov, O., Brodin, L., Sihra, T. S., Hvalby, O., Jensen, V., Zheng, D., McNamara, J. O., Greengard, P., and Andersen, P. (1995). Impairment of synaptic vesicle clustering and of synaptic transmission, and increased seizure propensity, in synapsin I-deficient mice. Proc. Natl. Acad. Sci. U.S.A. 92 9235-9239.

Li, Z., Burrone, J., Tyler, W. J., Hartman, K. N., Albeanu, D. F., and Murthy, V. N. (2005). Synaptic vesicle recycling studied in transgenic mice expressing synaptopHluorin. Proc. Natl. Acad. Sci. U.S.A. 102, 6131-6136.

Li,Z., and Murthy, V.N. (2001).Visualizing postendocytic traffic of synaptic vesicles at hippocampal synapses. Neuron 31, 593-605.

Lorteije, J. A. M., Rusu, S. I., Kushmerick, C., and Borst, J. G. G. (2009). Reliability and precision of the mouse calyx of Held synapse. J. Neurosci. 29, 13770-13784.

Mandell, J. W., Townes-Anderson, E., Czernik, A. J., Cameron, R., Greengard, P., and De Camilli, P. (1990). Synapsins in the vertebrate retina: absence from ribbon synapses and heterogeneous distribution among conventional synapses. Neuron $5,19-33$.

Maritzen, T., Podufall, J., and Haucke, V. (2010). Stonins- specialized adaptors for synaptic vesicle recycling and beyond? Traffic 11, 8-15.

Mathew, S. S., Pozzo-Miller, L., and Hablitz, J. J. (2008). Kainate modulates presynaptic GABA release from two vesicle pools. J. Neurosci. 28, 725-731.

Miesenbock, G., De Angelis, D. A., and Rothman, J. E. (1998). Visualizing secretion and synaptic transmission with $\mathrm{pH}$-sensitive green fluorescent proteins. Nature 394, 192-195.

Mitchell, S. J., and Ryan, T. A. (2004). Syntaxin-1A is excluded from recycling synaptic vesicles at nerve terminals. J. Neurosci. 24, 4884-4888.

Moser, T., Neef, A., and Khimich, D. (2006). Mechanisms underlying the temporal precision of sound coding at the inner hair cell ribbon synapse. J. Physiol. 576, 55-62.

Newton, A. J., Kirchhausen, T., and Murthy, V. N. (2006). Inhibition of dynamin completely blocks compensatory synaptic vesicle endocytosis. Proc. Natl. Acad. Sci. U.S.A. 103 , 17955-17960 
Nouvian, R., Beutner, D., Parsons, T. D., and Moser, T. (2006). Structure and function of the hair cell ribbon synapse. J. Membr. Biol. 209, 153-165.

Opazo, F., Punge, A., Buckers, J., Hoopmann, P., Kastrup, L., Hell, S. W., and Rizzoli, S. O. (2010). Limited intermixing of synaptic vesicle components upon vesicle recycling. Traffic 11, 800-812.

Parsons, T. D., and Sterling, P. (2003). Synaptic ribbon. Conveyor belt or safety belt? Neuron 37, 379-382.

Pechstein, A., Shupliakov, O., and Haucke, V. (2010). Intersectin 1: a versatile actor in the synaptic vesicle cycle. Biochem. Soc. Trans. 38, 181-186.

Petzold, G. C., Albeanu, D. F., Sato, T. F., and Murthy, V.N. (2008). Coupling of neural activity to blood flow in olfactory glomeruli is mediated by astrocytic pathways. Neuron 58, 897-910.

Pieribone, V. A., Shupliakov, O., Brodin, L., Hilfiker-Rothenfluh, S., Czernik, A. J., and Greengard, P. (1995). Distinct pools of synaptic vesicles in neurotransmitter release. Nature 375 , 493-497.

Pyle, J. L., Kavalali, E. T., Piedras-Rentería, E. S., and Tsien, R. W. (2000). Rapid reuse of readily releasable pool vesicles at hippocampal synapses. Neuron 28 , 221-231.

Rettig, J., and Neher, E. (2002). Emerging roles of presynaptic proteins in $\mathrm{Ca}^{2+}$ triggered exocytosis. Science 298, 781-785.

Richards, D.A., Guatimosim, C., and Betz, W. J. (2000). Two endocytic recycling routes selectively fill two vesicle pools in frog motor nerve terminals. Neuron 27, 551-559.

Richards, D. A., Guatimosim, C., Rizzoli, S. O., and Betz, W. J. (2003). Synaptic vesicle pools at the frog neuromuscular junction. Neuron 39, 529-541.

Rizzoli, S. O., Bethani, I., Zwilling, D., Wenzel, D., Siddiqui, T. J., Brandhorst, D., and Jahn, R. (2006). Evidence for early endosome-like fusion of recently endocytosed synaptic vesicles. Traffic 7, 1163-1176.

Rizzoli, S. O., and Betz, W. J. (2002). Effects of 2-(4-morpholinyl)-8phenyl-4H-1-benzopyran-4-one on synaptic vesicle cycling at the frog neuromuscular junction. J. Neurosci. $22,10680-10689$.

Rizzoli, S. O., and Betz, W. J. (2004). The structural organization of the readily releasable pool of synaptic vesicles. Science 303, 2037-2039.

Rizzoli, S. O., and Betz, W. J. (2005). Synaptic vesicle pools. Nat. Rev. Neurosci. 6, 57-69.

Rizzoli, S. O., and Jahn, R. (2007). Kissand-run, collapse and 'readily retrievable’ vesicles. Traffic 8, 1137-1144.
Roos, A., and Boron, W. F. (1981). Intracellular pH. Physiol. Rev. 61, 296-434.

Rosenmund, C., and Stevens, C. F. (1996). Definition of the readily releasable pool of vesicles at hippocampal synapses. Neuron 16, 1197-1207.

Royle, S. J., and Lagnado, L. (2010). Clathrin-mediated endocytosis at the synaptic terminal: bridging the gap between physiology and molecules. Traffic; accepted article; doi: 10.1111/j.1600-0854.2010.01104.x

Sankaranarayanan, S., De Angelis, D., Rothman, J.E., and Ryan, T.A. (2000). The use of pHluorins for optical measurements of presynaptic activity. Biophys. J. 79, 2199-2208.

Sara, Y., Virmani, T., Deak, F., Liu, X., and Kavalali, E. T. (2005). An isolated pool of vesicles recycles at rest and drives spontaneous neurotransmission. Neuron 45, 563-573.

Sätzler, K., Söhl,L.F., Bollmann, J.H., Borst, J. G., Frotscher, M., Sakmann, B., and Lübke,J.H. (2002). Three-dimensional reconstruction of a calyx of Held and its postsynaptic principal neuron in the medial nucleus of the trapezoid body. J. Neurosci. 22, 10567-10579.

Scheuss, V., Schneggenburger, R., and Neher, E. (2002). Separation of presynaptic and postsynaptic contributions to depression by covariance analysis of successive EPSCs at the calyx of held synapse. J. Neurosci. 22, 728-739.

Schneggenburger, R., Meyer, A. C., and Neher, E. (1999). Released fraction and total size of a pool of immediately available transmitter quanta at a calyx synapse. Neuron 23, 399-409.

Schneggenburger, R., and Neher,E. (2000). Intracellular calcium dependence of transmitter release rates at a fast central synapse. Nature 406, 889-893.

Schneggenburger, R., and Neher,E. (2005). Presynaptic calcium and control of vesicle fusion. Curr. Opin. Neurobiol. 15, 266-274.

Schneggenburger, R., Sakaba, T., and Neher, E. (2002). Vesicle pools and short-term synaptic depression: lessons from a large synapse. Trends Neurosci. 25, 206-212.

Shtrahman, M., Yeung, C., Nauen, D. W., Bi, G. Q., and Wu, X. L. (2005). Probing vesicle dynamics in single hippocampal synapses. Biophys. J. 89, 3615-3627.

Siksou, L., Rostaing, P., Lechaire, J. P., Boudier, T., Ohtsuka, T., Fejtova, A., Kao, H.T., Greengard, P., Gundelfinger, E. D., Triller, A., and Marty, S. (2007). Three-dimensional architecture of presynaptic terminal cytomatrix. $J$. Neurosci. 27, 6868-6877.

Staras, K., Branco, T., Burden, J. J., Pozo, K., Darcy, K., Marra, V., Ratnayaka,A., and
Goda, Y. (2010). A vesicle superpool spans multiple presynaptic terminals in hippocampal neurons. Neuron 66 37-44.

Sun, J. Y., Wu, X. S., and Wu, L. G. (2002). Single and multiple vesicle fusion induce different rates of endocytosis at a central synapse. Nature 417, 555-559.

Tabares, L., Ruiz, R., Linares-Clemente, P., Gaffield, M. A., Alvarez de Toledo, G., Fernandez-Chacon, R., and Betz, W. J. (2007). Monitoring synaptic function at the neuromuscular junction of a mouse expressing synaptopHluorin. J. Neurosci. 27, 5422-5430.

Takamori, S., Holt, M., Stenius, K., Lemke, E. A., Gronborg, M., Riedel, D., Urlaub, H., Schenck, S., Brugger, B., Ringler, P., Muller, S. A., Rammner, B., Grater, F., Hub, J. S., De Groot, B. L., Mieskes, G., Moriyama, Y., Klingauf, J., Grubmuller, H., Heuser, J., Wieland, F., and Jahn, R. (2006). Molecular anatomy of a trafficking organelle. Cell 127, 831-846.

Teng, H., Cole, J. C., Roberts, R. L., and Wilkinson, R. S. (1999). Endocytic active zones: hot spots for endocytosis in vertebrate neuromuscular terminals. J. Neurosci. 19, 4855-4866.

Teng, H., and Wilkinson, R. S. (2000). Clathrin-mediated endocytosis near active zones in snake motor boutons. J. Neurosci. 20, 7986-7993.

Valtorta, F., Jahn, R., Fesce, R., Greengard, P., and Ceccarelli, B. (1988). Synaptophysin (p38) at the frog neuromuscular junction: its incorporation into the axolemma and recycling after intense quantal secretion. J. Cell Biol. 107, 2717-2727.

Voglmaier, S. M., Kam, K., Yang, H., Fortin, D. L., Hua, Z., Nicoll, R. A., and Edwards, R. H. (2006). Distinct endocytic pathways control the rate and extent of synaptic vesicle protein recycling. Neuron 51, 71-84.

von Gersdorff, H., and Matthews, G. (1999). Electrophysiology of synaptic vesicle cycling. Annu. Rev. Physiol. 61 , 725-752.

Westphal, V., Rizzoli, S. O., Lauterbach, M. A., Kamin, D., Jahn, R., and Hell, S. W. (2008). Video-rate far-field optical nanoscopy dissects synaptic vesicle movement. Science 320, 246-249.

Wienisch, M., and Klingauf, J. (2006) Vesicular proteins exocytosed and subsequently retrieved by compensatory endocytosis are nonidentical. Nat. Neurosci. 9, 1019-1027.

Willig, K. I., Rizzoli, S. O., Westphal, V., Jahn, R., and Hell, S. W. (2006). STED microscopy reveals that synaptotagmin remains clustered after synaptic vesicle exocytosis. Nature 440, 935-939.

Wu, W., and Wu, L. G. (2007). Rapid bulk endocytosis and its kinetics of fission pore closure at a central synapse. Proc. Natl. Acad. Sci. U.S.A. 104, 10234-10239.

Wu, W., Xu, J., Wu, X. S., and Wu, L. G. (2005). Activity-dependent acceleration of endocytosis at a central synapse. J. Neurosci. 25, 11676-11683.

Wu, X. S., and Wu, L. G. (2009). Rapid endocytosis does not recycle vesicles within the readily releasable pool. J. Neurosci. 29, 11038-11042.

Wucherpfennig, T., Wilsch-Brauninger, M., and Gonzalez-Gaitan, M. (2003). Role of Drosophila Rab5 during endosomal trafficking at the synapse and evoked neurotransmitter release. $J$. Cell Biol. 161, 609-624.

Wyatt, R. M., and Balice-Gordon, R. J. (2008). Heterogeneity in synaptic vesicle release at neuromuscular synapses of mice expressing synaptopHluorin. J. Neurosci. 28, 325-335.

Yamashita, T., Hige, T., and Takahashi, T. (2005). Vesicle endocytosis requires dynamin-dependent GTP hydrolysis at a fast CNS synapse. Science 307, 124-127.

Yeung, C., Shtrahman, M., and Wu, X. L. (2007). Stick-and-diffuse and caged diffusion: a comparison of two models of synaptic vesicle dynamics. Biophys. J. 92, 2271-2280.

Zhang, Q., Li, Y., and Tsien, R. W. (2009). The dynamic control of kiss-andrun and vesicular reuse probed with single nanoparticles. Science 323, 1448-1453.

Zhu, Y., and Stevens, C. F. (2008). Probing synaptic vesicle fusion by altering mechanical properties of the neuronal surface membrane. Proc. Natl. Acad. Sci. U.S.A. 105, 18018-18022.

Zhu, Y., Xu, J., and Heinemann, S. F. (2009). Two pathways of synaptic vesicle retrieval revealed by single-vesicle imaging. Neuron 61, 397-411.

Conflict of Interest Statement: The authors declare the absence of any commercial or financial relationships that could be construed as a potential conflict of interest.

Received: 30 May 2010; paperpending published: 07 July 2010; accepted: 02 August 2010; published online: 05 October 2010. Citation: Denker A and Rizzoli SO (2010) Synaptic vesicle pools: an update. Front. Syn. Neurosci. 2:135. doi: 10.3389/ fnsyn.2010.00135

Copyright (C) 2010 Denker and Rizzoli. This is an open-access article subject to an exclusive license agreement between the authors and the Frontiers Research Foundation, which permits unrestricted use, distribution, and reproduction in any medium, provided the original authors and source are credited. 